\title{
47. A Canonical Form of a System of Microdifferential Equations with Non-Involutory Characteristics and Branching of Singularities
}

\author{
By Toshinori ÔAKU \\ Department of Mathematics, University of Tokyo \\ (Communicated by Kôsaku YosidA, M. J. A., April 13, 1981)
}

We study a system $\mathscr{M}$ of microdifferential (=pseudodifferential) equations. We assume that the characteristic variety $V$ of $\mathscr{M}$ is the union of two regular submanifolds with non-involutory intersection. We also assume that $\mathscr{M}$ has regular singularities along $V$. (Precise assumptions will be given below.) In $\S 1$, we give a canonical form of $\mathscr{M}$ in the complex domain. Applying this result, we study in $\S 2$ the branching of supports of microfunction solutions of $\mathscr{M}$ under the additional assumption that $\mathscr{M}$ is hyperbolic. Details of this article will appear elsewhere.

$\S 1$. A canonical form of a system with regular singularities along its non-involutory characteristics. Let $X$ be an $n$-dimensional complex manifold and $T^{*} X$ be its cotangent bundle. We identify the zero section of $T^{*} X$ with $X$. Let $z=\left(z_{1}, \ldots, z_{n}\right)$ be a local coordinate system of $X$. Then $(z,\langle\zeta, d z\rangle)=(z, \zeta)=\left(z_{1}, \cdots, z_{n}, \zeta_{1}, \cdots, \zeta_{n}\right)$ denotes a point of $T^{*} X$. We denote by $\mathcal{E}_{X}$ the sheaf on $T^{*} X$ of microdifferential operators (of finite order). Note that $\mathcal{E}_{X}$ is denoted by $\mathscr{Q}_{X}^{f}$ in [6]. Let $\mathcal{O}(j)$ be the sheaf on $T^{*} X$ of holomorphic functions homogeneous of degree $j$ with respect to the fiber coordinates. We denote by $\mathcal{E}(j)$ the sheaf of microdifferential operators of order at most $j$. There is a natural homomorphism

$$
\sigma_{j}: \mathcal{E}(j) \rightarrow \mathcal{O}(j) \cong \mathcal{E}(j) / \mathcal{E}(j-1) .
$$

If $P \in \mathcal{E}(j)-\mathcal{E}(j-1)$, we call $\sigma(P)=\sigma_{j}(P)$ the principal symbol of $P$. For a homogeneous (=conic) involutory analytic subset $V$ of $T^{*} X-X$, we set $I_{V}(j)=\left\{f \in \mathcal{O}(j) ;\left.f\right|_{V}=0\right\}$. Then $\mathcal{O}_{V}(0)=\mathcal{O}(0) / I_{V}(0)$ is a coherent sheaf of rings on $V$. We set $g_{V}=\left\{P \in \mathcal{E}(1) ; \sigma_{1}(P) \in I_{V}(1)\right\}$ and denote by $\mathcal{E}_{V}$ the subring of $\mathcal{E}_{X}$ generated by $\mathcal{g}_{V}$.

Let $\omega=\zeta_{1} d z_{1}+\cdots+\zeta_{n} d z_{n}$ be the fundamental 1-form on $T^{*} X$. A homogeneous involutory submanifold of $T^{*} X-X$ is said to be regular if the pull back of $\omega$ to it vanishes nowhere.

For a submanifold $W$ of $T^{*} X$ and a point $p$ of $W$, we say that $p$ is a point of rank $2 r$ in $W$ if the rank of the skew-symmetric bilinear form $d \omega$ on $T_{p} W$ is of rank $2 r$. If each point of $W$ is a point of rank $2 r$ in $W$, we say that $W$ is of rank $2 r$ and write rank $W=2 r$. 
Now let $\mathscr{M}$ be a coherent $\mathcal{E}_{X}$-module (i.e., a system of microdifferential equations) defined on an open subset $\Omega$ of $T^{*} X-X$; let $V=V_{1}$ $\cup V_{2}$ be a homogeneous involutory analytic subset of $\Omega$. We assume the following conditions:

(A.1) $V_{1}$ and $V_{2}$ are $d$-codimensional homogeneous rezular involutory submanifolds of $\Omega$, and $V_{0}=V_{1} \cap V_{2}$ is non-singular.

(A.2) $V_{1}$ and $V_{2}$ intersect normally, i.e., $T_{p} V_{1} \cap T_{p} V_{2}=T_{p} V_{0}$ for any $p \in V_{0}$.

(A.3) $\operatorname{dim} V_{1}=\operatorname{dim} V_{2}=\operatorname{dim} V_{0}+1$.

(A.4) $\operatorname{rank} V_{1}=\operatorname{rank} V_{2}=\operatorname{rank} V_{0}$.

(A.5) $\mathscr{M}$ has regular singularities along $V$; i.e., any coherent sub- $\mathcal{E}_{V}$-module of $\mathscr{M}$ that is defined on an open subset of $\Omega$ is coherent over $\mathcal{E}(0)$. (See [5], [3].)

Let $p_{0}$ be a point of $V_{0}$. We can find a neighborhood $U$ of $p_{0}$ and a coherent sub- $\mathcal{E}_{V}$-module $\mathscr{M}_{0}$ of $\left.\mathscr{M}\right|_{U}$ such that $\mathcal{E}_{X} \mathscr{M}_{0}=\left.\mathscr{M}\right|_{U}$. In view of (A.5), we see that $\overline{\mathscr{M}}_{0}=\mathscr{M}_{0} / \mathcal{E}(-1) \mathscr{M}_{0}$ is a coherent $\mathcal{O}_{V}(0)$-module. We make the additional assumption:

(A.6) $\quad \overline{\mathscr{M}}_{0}$ is a locally free $\mathcal{O}_{V}(0)$-module of rank $m$.

Let $p$ be an arbitrary point in $V_{0} \cap U$. Then (A.6) ensures that there exist generators $u_{1}, \cdots, u_{m}$ of $\mathscr{M}_{0}$ over $\mathcal{E}(0)$ in a neighborhood of $p$ whose residue classes are free generators of $\overline{\mathscr{M}}_{0}$ over $\mathcal{O}_{V}(0)$. In view of (A.1)-(A.4), we can find two microdifferential operators $P_{1}$ and $P_{2}$ in a neighborhood of $p$ such that $\sigma\left(P_{j}\right)=0$ on $V_{j}(j=1,2)$ and that the Poisson bracket $\left\{\sigma\left(P_{1}\right), \sigma\left(P_{2}\right)\right\}$ never vanishes on $V_{0}$. Let $P_{j}$ be of order $l_{j}$ and set $l=l_{1}+l_{2}$. Assumption (A.5) guarantees the existence of $A_{i j}$ $\in \mathcal{E}(l-1)(i, j=1, \cdots, m)$ defined near $p$ such that

$$
P_{1} P_{2} u_{i}=\sum_{j=1}^{m} A_{i j} u_{j} \quad(i=1, \cdots, m) .
$$

Setting

$$
a_{i j}(p)=\sigma_{l-1}\left(A_{i j}\right)(p) /\left\{\sigma\left(P_{1}\right), \sigma\left(P_{2}\right)\right\}(p),
$$

we define a polynomial $e_{12}$ in $\lambda$ by

$$
e_{12}\left(\lambda, p, \mathscr{M}_{0}\right)=\operatorname{det}\left(\lambda I_{m}+\left(a_{i j}(p)\right)_{1 \leqq i, j \leqq m}\right) ;
$$

here $I_{m}$ is the unit matrix of dezree $m$. We can easily see that $e_{12}$ is independent of the choice of operators $P_{1}$ and $P_{2}$, and generators $u_{1}, \cdots, u_{m}$ of $\mathscr{M}_{0}$ mentioned above. Thus $e_{12}\left(\lambda, p, \mathscr{M}_{0}\right)$ is well-defined for $p \in V_{0} \cap U$.

Remark. Interchanging $V_{1}$ and $V_{2}$, we can define $e_{21}$ in the same manner and have the relation

$$
e_{21}\left(\lambda, p, \mathscr{M}_{0}\right)=(-1)^{m} e_{12}\left(-\lambda-1, p, \mathscr{M}_{0}\right) .
$$

Let $\lambda=\lambda_{1}, \cdots, \lambda_{m}$ be the roots of the equation $e_{12}\left(\lambda, p_{0}, \mathscr{M}_{0}\right)=0$ in $\lambda$. For each complex number $\lambda$, we define a set $J(\lambda)$ by

$$
J(\lambda)=\left\{j \in\{1, \cdots, m\} ; \lambda_{j}-\lambda \in Z\right\} .
$$


Put $m(\lambda)=\# J(\lambda)$ (the cardinal number of $J(\lambda)$ ). Further, we set $J(0,1)=\left\{j \in J(0) ; \lambda_{j}<0\right\}, \quad J(0,2)=\left\{j \in J(0) ; \lambda_{j} \geqq 0\right\}$, and $m(0, i)=\# J(0, i)$ for $i=1,2$. Put

Note that

$$
\Lambda=\{\lambda \in C ;-1<\operatorname{Re} \lambda \leqq 0, J(\lambda) \neq \phi\} .
$$

$$
m=\sum_{\lambda \in A} m(\lambda), \quad m(0)=m(0,1)+m(0,2) .
$$

We use the notation $D=\left(D_{1}, \cdots, D_{n}\right)$ with $D_{j}=\partial / \partial z_{j}$ and set $z^{\prime}$ $=\left(z_{d+1}, \cdots, z_{n}\right)$ and $D^{\prime}=\left(D_{d+1}, \cdots, D_{n}\right)$.

On the above assumptions we have the following

Theorem 1. There exists a quantized contact transformation $\Phi$ associated with a local contact transformation $\varphi$ of $T^{*} X$ such that $\mathscr{M}$ is isomorphic to $\Phi(\mathcal{l})$ as an $\mathcal{E}_{X}$-module. Here $\mathcal{U}$ is a system defined on a neighborhood of $\varphi\left(p_{0}\right)=\left(0, d z_{n}\right)$ which takes the following form:

$$
\begin{gathered}
\Re=\bigoplus_{\lambda \in \Lambda} \mathcal{I}_{\lambda}, \\
\Re_{\lambda}:\left(z_{1} D_{1} I_{m(\lambda)}-A_{\lambda}\right) v_{\lambda}=D_{2} v_{\lambda}=\cdots=D_{d} v_{\lambda}=0 ;
\end{gathered}
$$

here $A_{\lambda}$ is an $(m(\lambda), m(\lambda))$ matrix of microdifferential operators of order at most 0 defined near $\left(0, d z_{n}\right)$, and $v_{\lambda}$ is a column vector of $m(\lambda)$ unknown functions. Moreover, $A_{\lambda}$ has the form $A_{\lambda}=A_{\lambda}\left(z^{\prime}, D^{\prime}\right)$ for $\lambda \neq 0$, while $A_{0}$ has the form

$$
A_{0}=\left[\begin{array}{ll}
A_{11}\left(z^{\prime}, D^{\prime}\right) & A_{12}\left(z^{\prime}, D^{\prime}\right) D_{1} \\
z_{1} A_{21}\left(z^{\prime}, D^{\prime}\right) & A_{22}\left(z^{\prime}, D^{\prime}\right)
\end{array}\right]
$$

with $A_{i j}$ being an $(m(0, i), m(0, j))$ matrix of microdifferential operators. In addition, all the eigenvalues of $\sigma_{0}\left(A_{\lambda}\right)\left(0, d z_{n}\right)$ equal $\lambda$ if $\lambda \neq 0$; all the eigenvalues of $\sigma_{0}\left(A_{11}\right)\left(0, d z_{n}\right)$ are -1 , and those of $\sigma_{0}\left(A_{22}\right)\left(0, d z_{n}\right)$ are 0 .

Remark. When $m=1$, this theorem has been proved by Kashiwara-Kawai-Oshima [4, Th. 3]. To prove Theorem 1, we use methods due to Kashiwara-Oshima [5].

§2. Branching of supports of microfunction solutions. Let $M$ be an $n$-dimensional real analytic manifold and $X$ be its complexification. We denote by $T_{M}^{*} X=\sqrt{-1} T^{*} M$ the conormal bundle of $M$ in $T^{*} X$. Let $\mathcal{C}_{M}$ denote the sheaf on $T_{M}^{*} X$ of microfunctions.

Let $\mathcal{M}$ be a coherent $\mathcal{E}_{X}$-module defined on an open subset $\Omega$ of $T^{*} X-X$; let $V=V_{1} \cup V_{2}$ be a homogeneous involutory analytic subset of $\Omega$. Let the assumptions (A.1)-(A.6) be satisfied. Moreover, we assume

(A.7) $V_{1}$ and $V_{2}$ are real ; i.e., $V_{j}$ is a complexification of the real analytic manifold $V_{j}^{R}=V_{j} \cap T_{M}^{*} X$ for $j=1,2$.

Let $p_{0}$ be a point in $V_{0}^{R}=V_{1}^{R} \cap V_{2}^{R}$ and let $\lambda_{1}, \cdots, \lambda_{m}$ be the roots of the equation $e_{12}\left(\lambda, p_{0}, \mathcal{M}_{0}\right)=0$ in $\lambda$. Then we also assume

(A.8) $\lambda_{j} \notin\{-1,-2, \cdots\} \quad$ for $j=1, \cdots, m$.

Theorem 2. Under the assumptions (A.1)-(A.8), we have 


$$
\mathcal{H}_{\operatorname{Com}_{X}}\left(\mathcal{M}, \Gamma_{V_{1}^{R}}\left(\mathcal{C}_{M}\right)\right)_{p_{0}}=0 .
$$

Moreover, if $\lambda_{j} \notin Z$ for $j=1, \cdots, m$, we have

$$
\mathcal{E}_{x t^{i}}^{i}\left(\mathcal{M}, \Gamma_{V_{k}}^{R}\left(\mathcal{C}_{M}\right)\right)_{p_{0}}=0
$$

for any $i \in Z$ and $k=1,2$.

Let us denote by $b_{j}\left(p_{0}\right)$ the bicharacteristic of $V_{j}^{R}$ through $p_{0}$ for $j$ $=1,2$. Assumptions (A.1)-(A.4) and (A.7) imply that $b_{1}\left(p_{0}\right)$ and $b_{2}\left(p_{0}\right)$ intersect normally and their intersection is 1-codimensional both in $b_{1}\left(p_{0}\right)$ and in $b_{2}\left(p_{0}\right)$. Hence $b_{j}\left(p_{0}\right)-b_{k}\left(p_{0}\right)=b_{j}\left(p_{0}\right)-V_{0}^{R}$ consists of two connected components $b_{j}^{+}\left(p_{0}\right)$ and $b_{j}^{-}\left(p_{0}\right)$ in a neighborhood of $p_{0}$ for $j, k$ $=1,2$ and $j \neq k$. Here the choice of $b_{j}^{+}\left(p_{0}\right)$ and $b_{j}^{-}\left(p_{0}\right)$ is arbitrary.

Theorem 3. Suppose that (A.1)-(A.8) are satisfied. Then there exists a fundamental neighborhood system $\left\{U_{l}\right\}_{l=1,2, \ldots}$ of $p_{0}$ in $T_{M}^{*} X$ which satisfies the following: If $f$ is a microfunction solution of $\mathscr{M}$ (i.e., a section of $\left.\mathscr{H}_{\mathrm{om}}\left(\mathscr{M}, \mathcal{C}_{M}\right)\right)$ defined on some $U_{l}$ such that

$$
b_{2}^{+}\left(p_{0}\right) \cap U_{l} \not \subset \operatorname{supp} f, \quad\left(b_{1}\left(p_{0}\right) \cup b_{2}^{-}\left(p_{0}\right)\right) \cap U_{l} \not \subset \operatorname{supp} f,
$$

then $f$ vanishes on a neighborhood of $\left(b_{1}\left(p_{0}\right) \cup b_{2}\left(p_{0}\right)\right) \cap U_{l}$.

For a subset $S$ of $T_{M}^{*} X$, we set $\boldsymbol{R}^{+} S=\{c p ; c \in R, c>0, p \in S\}$.

Theorem 4. Let $\mathscr{M}$ and $V$ satisfy (A.1)-(A.7). Then there exist a neighborhood $U^{\prime}$ of $p_{0}$ in $T_{M}^{*} X$ and a microfunction solution $f$ of $\mathscr{M}$ defined on $U^{\prime}$ such that

$$
\boldsymbol{R}^{+} b_{1}\left(p_{0}\right) \cap U^{\prime} \subset \operatorname{supp} f \subset \boldsymbol{R}^{+}\left(b_{1}\left(p_{0}\right) \cup b_{2}^{+}\left(p_{0}\right)\right) \cap U^{\prime} .
$$

Moreover, if $\lambda_{j} \notin\{-1,-2, \cdots\}$ for some $j \in\{1, \cdots, m\}$, we can choose $f$ so that

$$
\operatorname{supp} f=\boldsymbol{R}^{+}\left(b_{1}\left(p_{0}\right) \cup b_{2}^{+}\left(p_{0}\right)\right) \cap U^{\prime} .
$$

Corollary. Let $P_{1}$ and $P_{2}$ be microdifferential operators of order $l_{1}$ and $l_{2}$ respectively, defined on a neighborhood of $p_{0} \in T_{M}^{*} X-M$. Suppose that

$$
\sigma\left(P_{1}\right)\left(p_{0}\right)=\sigma\left(P_{2}\right)\left(p_{0}\right)=0, \quad\left\{\sigma\left(P_{1}\right), \sigma\left(P_{2}\right)\right\}\left(p_{0}\right) \neq 0,
$$

and that $\sigma\left(P_{1}\right)$ and $\sigma\left(P_{2}\right)$ are real valued on $T_{M}^{*} X$. Let $A_{i j}(i, j=1, \cdots$, $m)$ be microdifferential operators of order at most $l-1=l_{1}+l_{2}-1$ defined near $p_{0}$, and set $A=\left(A_{i j}\right)$. We assume that no eigenvalue of the matrix $\left(\sigma_{l-1}\left(A_{i j}\right)\left(p_{0}\right) /\left\{\sigma\left(P_{1}\right), \sigma\left(P_{2}\right)\right\}\left(p_{0}\right)\right)$ is a negative integer. Then the conclusions of Theorems 3 and 4 are valid for the system

$$
\left(P_{1} P_{2} I_{m}+A\right) u=0
$$

with $V_{k}=\left\{\sigma\left(P_{k}\right)=0\right\}$ for $k=1,2$. Here $u$ is a column vector of $m$ unknown functions.

Remark. When $m=1$, an analogue in the $C^{\infty}$-category of this corollary has been obtained by Ivrii [2] and Hanges [1].

Example. Set $x=\left(x_{1}, \cdots, x_{n}\right) \in \boldsymbol{R}^{n}$ and $D_{j}=\partial / \partial x_{j}$. We put

$$
P=\left(D_{1}^{2}-x_{1}^{2}\left(D_{2}^{2}+\cdots+D_{n}^{2}\right)\right) I_{m}+Q ;
$$

here $Q$ is an $(m, m)$ matrix of microdifferential operators of order at most 1 defined on a neighborhood of $p_{0}=\left(0, \sqrt{-1} d x_{n}\right) \in \sqrt{-1} T^{*} R^{n}$. 
Set $b_{k}\left(p_{0}\right)=\left\{(x, \sqrt{-1} \xi) \in \sqrt{-1} T^{*} R^{n} ; x_{n}=(-1)^{k} x_{1}^{2} / 2, x_{2}=\cdots=x_{n-1}=0\right.$, $\left.\xi_{1}=-(-1)^{k} x_{1}, \xi_{2}=\cdots=\xi_{n-1}=0, \xi_{n}=1\right\}$ and $b_{k}^{ \pm}\left(p_{0}\right)=\left\{(x, \sqrt{-1} \xi) \in b_{k}\left(p_{0}\right)\right.$; $\left.\pm x_{1}>0\right\}$ for $k=1,2$. Assume that no eigenvalue of the matrix $\sigma_{1}(Q)\left(p_{0}\right)$ belongs to $\{ \pm \sqrt{-1}, \pm 3 \sqrt{-1}, \pm 5 \sqrt{-1}, \cdots\}$. Let $f$ be a column vector of $m$ microfunctions defined on a neighborhood of $p_{0}$ such that $P f=0$. Under these assumptions, if $f$ vanishes on any two of half-bicharacteristics $b_{1}^{+}\left(p_{0}\right), b_{1}^{-}\left(p_{0}\right), b_{2}^{+}\left(p_{0}\right)$, and $b_{2}^{-}\left(p_{0}\right)$, then $f$ vanishes on a neighborhood of $p_{0}$.

\section{References}

[1] N. Hanges: Parametrices and propagation of singularities for operators with non-involutive characteristics. Indiana Univ. Math. J., 28, 87-97 (1979).

[2] V. Ya. Ivrii: Wave fronts of solutions of certain pseudodifferential equations. Funct. Anal. Appl., 10, 141-142 (1976).

[ 3 ] M. Kashiwara and T. Kawai: On holonomic systems of micro-differential equations, III (to appear).

[4] M. Kashiwara, T. Kawai, and T. Oshima: Structure of cohomology groups whose coefficients are microfunction solution sheaves of systems of pseudodifferential equations with multiple characteristics. I. Proc. Japan Acad., 50, 420-425 (1974).

[5] M. Kashiwara and T. Oshima: Systems of differential equations with regular singularities and their boundary value problems. Ann. of Math., 106, 145-200 (1977).

[ 6 ] M. Sato, T. Kawai, and M. Kashiwara: Microfunctions and pseudo-differential equations. Lect. Notes in Math., vol. 287, Springer, Berlin-HeidelbergNew York, pp. 265-529 (1973). 